\title{
Using misconceptions research in the design of optics instructional materials and teacher professional development programs
}

Stephen Pompea, Erin Dokter, Constance Walker, Robert Sparks

Stephen M. Pompea, Erin F. Dokter, Constance E. Walker, Robert T. Sparks, "Using misconceptions research in the design of optics instructional materials and teacher professional development programs," Proc. SPIE 9665, Tenth International Topical Meeting on Education and Training in Optics and Photonics, 966515 (3 June 2007); doi: 10.1117/12.2207517

SPIE Event: Tenth International Topical Meeting on Education and Training in Optics and Photonics, 2007, Ottawa, Ontario, Canada 


\title{
Using Misconceptions Research in the Design of Optics Instructional Materials and Teacher Professional Development Programs
}

\author{
Stephen M. Pompea ${ }^{1}$, Erin F. Dokter ${ }^{2}$, Constance E. Walker ${ }^{1}$, and Robert T. Sparks ${ }^{1}$ \\ 1. National Optical Astronomy Observatory, 950 N. Cherry Avenue, Tucson, Arizona, 85719 USA \\ 2. Conceptual Astronomy and Physics Research (CAPER) Team, Steward Observatory, University of Arizona, Tucson Arizona 85721 USA \\ Author Contact: spompea@noao.edu Voice 520.318.8285
}

\begin{abstract}
To create the Hands-On Optics program and its associated instructional materials, we needed to understand a number of basic optics misconceptions held by children (and adults) and how to address them through a proper educational approach. The activities have been built with an understanding of the naïve concepts many people have about light, color, and optical phenomena in general. Our own experience is that the concepts that children and adults have of light are often not that different from each other. This paper explores the most common misconceptions about light and color, according to educational research, and describes how they can be addressed in optics education programs. This understanding of misconceptions was useful as well in the professional development component of the program where educators were trained on the Hands-On Optics modules. The professional development work for the optics industry volunteers who worked with the educators was also based on research on how an optics professional can work more effectively in multi-cultural settings-an area with great applicability to industry volunteers working in the very different culture of science centers or after-school programs.
\end{abstract}

Keywords: optics education, professional development, inquiry, misconceptions

\section{Introduction}

The Hands-On Optics (HOO) is a National Science Foundation-funded program designed to address the disconnect between the ideas held by young children about light and basic optical concepts (Pompea et al. 2005). The program works to instill a sensible conceptual foundation for a limited number of optics concepts. The HOO materials are partially based on previous inquiry-based materials developed through the Lawrence Hall of Science Great Explorations in Math and Science program. These materials (e.g. the Invisible Universe book) explore the electromagnetic spectrum using inquiry (Pompea and Gould 2003; Pompea and Gek 2002). In a survey of topics appropriate for formal optics education (Pompea and Stepp 1995) and for informal education programs (Pompea and Hawkins 2002) a number of optics areas of particular educational interest were identified. However, the proper approach to these topics can be complicated and must rely on an understanding of naïve theories held by many students.

Unfortunately, viewing the field of optics from an optical expert's point of view does not always serve the educational process. One must understand how a novice approaches light and color and appreciate the perspective a child brings to learning about optics. A student does not reason like a scientist and prior knowledge may impede progress in learning the key concepts. Novices differ from experts in that novices do not notice meaningful patterns in a given field of study. Novices do not have the organizational structure of the content knowledge that an expert possesses. The knowledge of an expert has a sense of context or conditions; it is not a set of facts, propositions, or theorems. Experts are very flexible in their thinking processes and also have the ability to retrieve important knowledge sets with little efforts. Their knowledge is ingrained in them rather than attached to them and they have an intuitive feel for their subject.

This paper is an attempt to consider briefly what research on human learning tells us about this transition from novice to expert and how an understanding of optics misconceptions and approaches to dealing with them can guide us in the design of instructional materials.

Tenth International Topical Meeting on Education and Training in Optics and Photonics, edited by Marc Nantel, Proc. of SPIE Vol. 9665, 966515 • @ 2007 SPIE, OSA, IEEE, ICO doi: $10.1117 / 12.2207517$ 


\section{Background}

A program such as Hands-On Optics must directly address common misconceptions that students (and educators) have about basic optics principles such as reflection, the nature of light, and how images are formed. Research on misconceptions reinforces the notion that misconceptions among students are the rule rather than the exception. Some of the common misconceptions include not viewing light as something that travels but rather only as its source (the Sun, a light bulb, etc.). This leads to difficulty in understanding the formation of shadow and the direction they take. The research shows that middle-school students (ages 10-13) understand that mirrors can reflect light. However, they often reject the idea that everyday objects also reflect light, according to Guesne (1985) and Ramadas and Driver (1989). Similarly, many elementary and middle school students do not realize that their eyes receive light when they look at an object. The notion that the eyes generate light that radiates outwards is a common one. These students therefore have quite a varied set of conceptions about how vision works. Some 5thgraders, though, can understand seeing as "detecting" reflected light after specially designed instruction (Anderson \& Smith, 1983) For more details on optics concepts such as waves see the Atlas of Science Literacy. This volume uses concepts maps to understand the relationship between a number of key science concepts. For additional study, Driver et al. (1994), Stepans (1996), and Comins (2001) all have excellent discussions of misconceptions.

\section{Approaches to Dealing with Misconceptions: Conceptual Change Models}

A number of approaches to dealing with misconceptions have been proposed. One of the more practical perspectives is provided by Stepans (1996) who proposed a conceptual change model to help students become aware of their misconceptions and examine their validity. Stepans' model has six steps:

1. Students become aware of their own preconceptions about a concept by thinking about it and making predictions (committing to an outcome) before any activity begins.

2. Students expose their beliefs by sharing them, initially in small groups and then with the entire class.

3. Students confront their beliefs by testing and discussing them, initially in small groups and then with the entire class.

4. Students work toward resolving conflicts (if any) between their ideas (based on the revealed preconceptions and class discussion) and their observations, thereby accommodating the new concept.

5. Students extend the concept by trying to make connections between the concept learned in the classroom and other situations, including their daily lives.

6. Students are encouraged to go beyond, pursuing additional questions and problems of their choice related to the concept.

Another step that can be done even before these steps is for the educator or educational designer to do a "front end" study of the naïve theories and misconceptions of the audience. This can best be done through careful interviewing of the audience in a supportive, low-stress setting. With thoughtful in-depth questioning and especially with attentive listening a significant amount of information can be obtained. Nearly all museum exhibitions employ frontend studies to help the designer understand the knowledge base of the expected audience.

This type of interviewing technique was used successfully in the Harvard Private Universe Project (Schneps and Sadler 1989) that unveiled serious misconceptions about basic science area (such as the seasons, or simple electric circuits) from college graduates as well as children. Sadler (2000) explored how student concepts of light and color can change. To understand and measure conceptual development, Bardar et al. (1996) developed a light and spectroscopy concept inventory test that is now being widely used. These research results have informed and continue to inform our development process for Hands-On Optics materials and professional development. In our professional development program we train after-school educators and science center educators on the HOO modules and concepts. Most often the professional background of these educators is not strong in science or in science education.

\section{Common Naïve Conceptions about Light}

In the Hands-On Optics project we explicitly address a number of misconceptions or myths about light, building on the work done in the American Institute of Physics Operation Physics project (1988). We describe below fifteen commonly held beliefs and how they are approached in the HOO modules. The excerpts below constitute advice for museum and after-school educators, and optics industry volunteers, on how to address these selected myths. 
Myth 1: Light only reflects off mirrors and other smooth surfaces.

Many people believe you need a smooth surface for reflection to take place. This misconception is reinforced by our use of language. We say that you see your reflection in a mirror. Since you don't see your reflection in other surfaces, many people assume that light doesn't reflect off those surfaces. You may wish to start by showing your students the plane mirror. Ask them what they see in the mirror. Once your students agree they see their reflection in the mirror, ask them to look at a table or wall. Ask them if light reflects off the table or wall. This question may start a lively discussion about reflection.

The green laser is a nice demonstration tool to illustrate reflection. Point the green laser at a table or wall. It may help to dim the lights. You can see the laser does reflect off these surfaces! You may hold up a piece of paper or screen to help see the reflections.

Ask the students what they notice about the shape of the reflections. The laser produces a nice sharp dot on the surface. The reflection, however, is very spread out. Ask students what the difference is between the laser reflecting off of a mirror versus the laser reflecting off of a table or wall. Remind the students of the difference between specular and diffuse reflection. Reflection off the mirror is specular, where reflection off of the rough surfaces is diffuse. Remember the law of reflection always holds - for both smooth and rough surfaces.

Try other surfaces around the room. Coins can produce interesting reflections. Try to produce reflections off books, backpacks, or anything else you can think of.

\section{Myth 2: Objects are black because they do not reflect any light.}

In many textbooks, black is referred to as the absence of light. Many people assume that black objects do not reflect light. Take the glossy black plastic plate included in the kit. Ask the students if they think the black plate reflects light. Have them look at the plate and ask them if they can see their reflection. Discuss whether or not this implies the plate reflects light.

Next, take the green laser and point it at the glossy side of the black plate. Ask the students if they see a reflection from the plate. If the black plate does not reflect light, how can it cause a reflection? The black plate does reflect light. It is enough for you to see a reflection of yourself or the laser. Ask the students if the rough side of the plate will reflect light. They cannot see their own reflections in the rough side. Shine the laser onto the rough side of the plate. Ask them if they see a reflection and if how the rough side is different than then shiny side.

The rough side of the place also reflects a significant amount of the incoming light. Light incident on the rough side undergoes diffuse reflection as you can see from the spread out nature of the reflection.

\section{Myth 3: If you are five feet tall, you need a five-foot tall mirror to see your entire body at once.}

You have a 12"x12" mirror in the kit. If the above statement is true, you should only be able to see 12" of your face at one time. Have a student hold a ruler next to the face and look into the mirror (it is important that the mirror be as close to vertical as possible). Does the ruler just fill the mirror, or can the student see more?

Next, give the student a meter stick or tape measure. Have the student hold the meter stick next to their face and measure how much of the meter stick can be seen at one time. The student will probably see about two feet of the meter stick, or twice the size of the mirror!

Since we can see an object twice the size of the mirror, we can conclude you need a mirror only one half your height to see your entire body at once. Using ray diagrams, it is easy to show that a mirror must be a minimum of $1 / 2$ the person's height in order to see the image as a whole person.

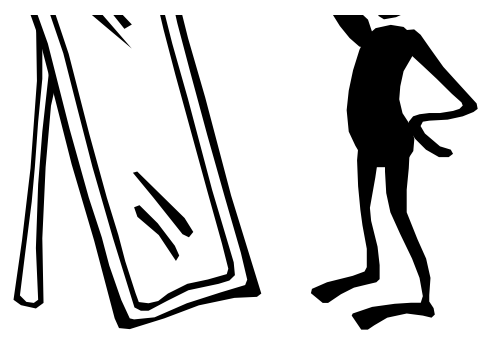
However, ray diagrams just aren't intuitive for the kids. The demonstration described above should help. 
You may have some students claim they cannot see their entire body in certain mirrors that seem large enough - for instance, a bathroom mirror above a sink. The sink may block the view of their feet. They may also have problems if the mirror is mounted too low or too high. The top of the mirror should be lined up roughly with the top of the head if you are truly going to see yourself in a mirror only one half your height!

Myth 4: You can see more of yourself if you move farther away from a mirror.

Many people believe that if you move farther away from a mirror, you can see more of yourself in the mirror, although this directly contradicts the idea that you need a mirror as tall as yourself to see your entire reflection!

Place the 12"x12" mirror vertically on a wall. Ask the students if and how they can use the mirror to see their entire body at once without moving the mirror. They will invariably try to move farther away (a crafty student may take the mirror off the wall and look into it at a steep angle, but this is outside the bounds of the question...more on this later).

Students will find that they cannot see their whole body at once! In the previous example, students saw the amount of their bodies they could see was twice the height of the mirror. Now they will see that this ratio holds even if they move closer to or farther away from the mirror.

If the students are still skeptical, try this. Have a student stand in front of the mirror. Tell the student to draw a circle on the mirror around his face with a dry erase or other washable marker. Have the student slowly back up and observe how the size of his or her face changes with respect to the size of the circle. The student will see that his or her face always takes up the same amount of space on the mirror!

Finally, place the mirror flat on the floor. Have a student stand close to (but not on top of!) the mirror. The student will be able to see almost the entire reflection (save for a small portion near their feet). Why they can see their entire body in this configuration? Nearly every part of your body sends light toward the mirror at a very large incident angle (remember, this is measured relative to the normal line).

Myth 5: Light stays on a mirror during reflection (light doesn't travel).

Many students believe that light stays on the surface of a mirror during a reflection. Light reflects off a mirror to your eyes. You do not see an image of an object unless the light enters your eyes. You can show light reflecting off a mirror with a flashlight and paper. Shine the light on the mirror. Hold the paper so you see the reflection. Move the paper closer and farther away from the mirror. You always see light on the paper. The light must leave the surface of the mirror to travel to the paper.

Myth 6: The image you see forms on the surface of the mirror.

Students see an image in a mirror and assume the image is on the surface of the mirror. The concept of a virtual image comes into play here. A virtual image is formed by diverging light rays and forms BEHIND the mirror. Virtual images cannot be projected onto a screen like real images. It is very counterintuitive to say an image forms behind the mirror as no light can travel through the mirror.

One way to counter this belief is to have a student look in a mirror. Have the student move away from the mirror. What happens to the reflection? The reflection appears to move away from the mirror in the opposite direction. The virtual image is where the student's reflection appears. The image appears behind the mirror, even though we know all light reflects from the surface of the mirror.

\section{Myth 7: An object is "seen" because light shines on it.}

Light can shine on an object but it may be invisible (i.e., we can't see it). Try this. Take a Pyrex test tube. Fill a beaker with Wesson Oil. Put the Pyrex test tube in the Wesson Oil and watch it disappear!

How can this be? There is clearly light shining on the test tube. However, no light is being reflected from the test tube. In order to see an object,

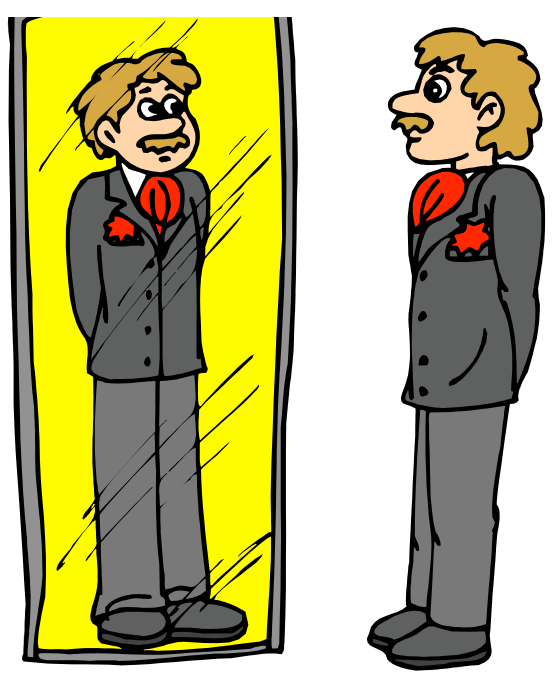


light must reflect off the object and enter your eye. If there is no light reflected, you may not be able to see the object.

This demonstration works because Wesson Oil and Pyrex have the same index of refraction. If two substances have the same index of refraction, no light is reflected at the boundary between the two objects. If you put the Pyrex test tube in tap water, it will be clearly visible.

\section{Myth 8: Mirrors reflect all light that shines on their surfaces.}

No mirror reflects $100 \%$ of the light that shines on it. Good mirrors reflect $95 \%$ of the light that is incident on them. The remaining $5 \%$ is absorbed and converted to heat. If you use a very intense light source, such as the Sun on a hot summer day, you can feel the mirror heat up from the energy it absorbs.

If students make careful observations during the activity in Module 2 called "Making a Dollar Out of a Penny." They may notice that the pennies that appear farther away are also dimmer. It takes more reflections to make the pennies that appear farther away. Each reflection causes some light to be lost and each new penny appears slightly dimmer. Astronomers are very concerned about light lost during the reflection process. Astronomical telescopes use special coatings on their mirrors and lenses to try and minimize the amount of light lost by reflecting off the mirrors in a telescope.

\section{Myth 9: Light always travels in a straight line.}

By now, students should have seen at least two examples that show light does not always travel in a straight line. They have seen light reflect off mirrors in Module 1 and observed light refract when it passes from air to the acrylic block in Module 3.

Light does travel in a straight line when it is traveling in a uniform medium. The direction light travels changes when the medium through which it travels changes. This change in medium can cause reflection, refraction, or even absorption of the light. Through these modules, students have only seen sudden changes in the direction of light, such as when light reflects off of a mirror or when it travels from air to plastic. The change in index of refraction can also be gradual, causing the path of light to gently curve. An example of this phenomenon is when sunlight encounters Earth's atmosphere. The upper layers of the atmosphere are very thin and have a lower index of refraction. The lower layers of the atmosphere are thicker and have a higher index of refraction. This changing index of refraction causes the path of the sunlight to curve.

An interesting consequence of this is that refraction slightly alters the time of sunrise and sunset. Refraction will make the Sun appear about half a degree higher in the sky than it really is (34 arc minutes on average). Therefore, the Sun appears to rise a few minutes earlier and set a few minutes later than it would if Earth had no atmosphere!

\section{Myth 10: Light travels infinitely fast.}

Light travels fast, but it has a finite speed just like everything else. Light in a vacuum travels at $3 \mathrm{X} 10^{8}$ meters per second. The speed of light in a vacuum is sometimes called the speed limit of the universe. Direct evidence that light has a finite speed is difficult, but not impossible to illustrate. Another interesting way to see the effect of the speed of light is using satellite television. Set up two televisions side by side. Have one of the televisions connected to an antenna. Connect the other television to cable or a satellite dish. Tune them both to the same local channel. You will notice that the television connected to the antenna receives the signal first!

What's going on in this case? Cable or satellite television bounces the signal to a satellite in geosynchronous orbit about 22,000 miles above the Earth's surface. The signal for cable or satellite television must travel to the satellite and back - meaning it gets to the television later due because it travels a greater distance.

\section{Myth 11: You can use a telescope to magnify objects as much as you desire.}

Many people believe you can always increase the magnification of a telescope. It is not uncommon to see advertisements for telescopes with diameters as small as 2 inches that claim to operate at 575 power!

Small telescopes cannot achieve such large magnifications for a variety of reasons. Even assuming perfect optics, a telescope is limited by its resolution or resolving power. Resolution is the ability of a telescope to see fine detail and 
separate closely spaced objects. Resolution depends primarily on the diameter of the telescope. If you try to magnify an object beyond the resolution of a telescope, you get a dim, fuzzy image.

A general rule of thumb for astronomers is that the maximum useful magnification for a telescope is about 50X (50 power) per inch of aperture. Therefore, a 2 inch diameter telescope generally cannot magnify more than about $100 \mathrm{X}$. The 50X per inch of aperture assumes you have very good optics (not always the case in inexpensive telescopes) and that the atmospheric seeing is very good.

The atmospheric seeing is another limiting factor in telescope resolution. If you have ever looked at hot pavement on a summer day, you have probably seen "heat waves." The heat waves are due to the fact that the index of refraction of air is very temperature-dependent. As the pavement heats up, the hot air above the pavement rises and causes turbulence. As light passes through air of different temperatures, its path is changed, leading to the heat waves.

Earth's atmosphere has a similar effect on light from the stars and planets. You can see this effect in the twinkling of stars. Even on a relatively calm night, a telescope will magnify any distortion present in the atmosphere. The best observing sites in the world rarely have seeing much better than one-half to one arcsecond (one arcsecond is $1 / 3600^{\text {th }}$ of a degree). More common observing sites have 2 to 3 arcsecond seeing or worse.

The Hubble Space Telescope was launched to get above the Earth's atmosphere. Although the Hubble has a relatively modest sized 2.5-meter diameter primary mirror, it does not have to look through Earth's atmosphere, so it yields much sharper views. The Hubble Space Telescope has a resolution of about 0.1 arc seconds, 10 times sharper than is typically possible from the ground.

In recent years, great advances have been made in overcoming the effects of atmospheric seeing through a process called adaptive optics (AO). Adaptive optics systems work by observing a star to precisely measure the distortions caused by Earth's atmosphere. Once the distortions are measured, they can be removed by quickly and precisely changing the shape of a small, flexible mirror in the telescope. Ground-based telescopes can produce images as good as the Hubble Space Telescope using adaptive optics systems.

Myth 12: An image is always formed at the focal point of the lens.

The focal point of a lens is where light rays that start out parallel will converge and form an image. Many physics books state that light rays from a distant object are parallel. While the light rays are not truly parallel, they are moving apart very slowly and will converge very close to the focal point.

For an object that is close to a lens or mirror, the incoming light rays are traveling in very different directions. Since the light rays are not close to parallel, they will not converge at the focal point. You can find where they will

converge through careful ray tracing or by using the equation

where $f$ is the focal length of the lens, $O$

is the object distance and $I$ is the image distance from the lens.

Myth 13: Polarizing filters are just dark plastic or glass.

A single ideal polarizing filter reduces the intensity of the transmitted light by $50 \%$. Looking through a polarizing filter leads many people to conclude they are looking through an ordinary, gray filter. Gray filters reduce the intensity of light by absorbing light. Gray filters absorb light that is polarized in all orientations. If a gray filter absorbs half of the vertically polarized light, it will also absorb half of the horizontally polarized light. If unpolarized light enters a gray filter, the transmitted light is also unpolarized.

A polarizing filter, on the other hand, absorbs all light except for one particular orientation. A polarizing filter can absorb all the vertically polarized light and let through the horizontally polarized light. If unpolarized light falls on a polarizing filter, the intensity of the transmitted light will be $50 \%$, just like in the previous examples. In this case, the transmitted light will be polarized. Some sunglasses are polarized and some are not. Unpolarized sunglasses can reduce the intensity of sunlight and protect your eyes from harmful UV radiation. Polarized sunglasses help reduce the glare, especially from horizontal surfaces such as roads or lakes. However, unpolarized sunglasses will not block glare from reflected light. 


\section{Myth 14: All radiation is harmful.}

Many people hear the word "radiation" and think of radiation poisoning or cancer. However not all radiation is harmful. Visible light is a form of electromagnetic radiation and we need to use it every day to see. Radio waves have very low energy and are generally considered harmless.

$\mathrm{X}$-rays and gamma rays are called ionizing radiation. Ionizing radiation can penetrate the skin and cause cell damage. However, even X-rays and gamma rays can be useful in certain medical therapies if used correctly. Ultraviolet light can cause sunburn and long-term exposure can cause skin cancer. Radiation can be harmful, but there are many types of harmless radiation and it is often useful in a wide variety of applications.

\section{Myth 15: Lasers emit tight, parallel beams of light.}

Most lasers are manufactured to produce a thin beam of light. We have come to expect lasers to look like this due to their portrayal in popular medial such as movies. Lasers are point sources and emit waves that spread out in a circular pattern as all other point sources do. Imagine dropping a rock in a pond and watching the waves spread out. This picture is close to a 2-D wave from a point source. In order to imagine a 3-D wave, you have to imagine spherical waves spreading out instead of circular waves.

So how we make lasers into thin beams? There are two ways this can be accomplished, both of which use techniques mentioned in Hands-On Optics modules. If you place a point source at the focal point of a lens, the lens will create a parallel beam of light. You can achieve the same effect by placing the point source at the focal point of a mirror. It is actually the lens or mirror that makes laser light into a parallel beam.

\section{Conclusion}

Misconceptions and naïve theories held by students are valuable to the educator and educational designer. To treat students as tabulae rasae would be serious mistake. An understanding of research on misconceptions and conceptual change has been extremely valuable to the Hands-On Optics project and in the creation of our materials and related programs. An understanding of common optics misconceptions provides an assessment not only of the knowledge base of the student but also on the basic structure of that knowledge. An understanding of this foundational knowledge is critical to our approach, much as it is in the construction of a building on a firm foundation. The underlying student knowledge base may have to be dismantled. If gaps are present in an otherwise sound base it may able to be rebuilt with an understanding of how conceptual change takes place.

Without this understanding of a student's knowledge base, a proper program in optics education cannot be designed. This is because new knowledge obtained through an education program will often be overlaid on top of the older, well-established, personal theories of how things work. In extreme cases, the new knowledge will be rejected because it does not conform to the previous (but wrong) knowledge that the student believes is solid. Often this old knowledge seems to adequately explain natural phenomena. Only by employing a conceptual change process can the required cognitive dissonance be created to allow the newer (correct) knowledge to find a stable niche or attachment point.

\section{References}

The American Association for the Advancement of Science (AAAS), Atlas of Science Literacy. Washington, DC: AAAS and National Science Teachers Association (co-publishers) See "Waves" map, p. 65 (2001).

C. Anderson, and E. Smith, Children's Conceptions of Light and Color: Understanding the Concept of Unseen Rays. East Lansing: Michigan State University (1983).

E. Bardar, E. Prather, K. Brecher, and T. Slater, "Development and Validation of the Light and Spectroscopy Concept Inventory", Astronomy Education Review Issue 2, Volume 5:103-113, (2006). 
N. F. Comins, Heavenly Errors: Misconceptions About the Real Nature of the Universe. New York: Columbia University Press (2001).

R. Driver, A. Squires, P. Rushworth, and V. Wood-Robinson, Making Sense of Secondary Science: Research Into Children's Ideas, Ch 17 Light, London: Routledge, (1994).

E. Guesne, “Light”, in R. Driver, E. Guesne, and A. Tiberghien (Eds.), Children's Ideas in Science (pp. 10-32). Milton Keynes, UK: Open University Press (1985).

Operation Physics, American Institute of Physics 1825 Connecticut Ave. NW, Suite 213 Washington, DC (1988) see http://www.aip.org or http://amasci.com/miscon/opphys.html

S. M. Pompea and T. K. Gek, "Optics in the Great Exploration in Math and Science (GEMS) Program: A Summary of Effective Pedagogical Approaches", Proceedings of the SPIE: Education and Training in Optics and Photonics, 4588 (2002).

S. M. Pompea and A. Gould, Invisible Universe: The Electromagnetic Spectrum from Radio Waves to Gamma Rays, Great Explorations in Math and Science (GEMS) Series, Lawrence Hall of Science, Berkeley, CA. (2003).

S. M. Pompea and I. Hawkins, "Increasing Science Literacy in Optics and Photonics through Science Centers, Museums, and Web-based Exhibits", Proceedings of the SPIE: Education and Training in Optics and Photonics, 4588 (2002).

S. M. Pompea, A. Johnson, E. Arthurs and C. E. Walker. "Hands-On Optics: An Educational Initiative for Exploring Light and Color in After-School Programs, Museums, and Hands-On Science Centers", Proceedings, Ninth International Topical Meeting on Education and Training in Optics and Photonics, Marseille, France. (2005)

S. M. Pompea and L. Stepp, "Great Ideas for Teaching Optics", Proceedings SPIE: International Conference on Education in Optics, Edited by M. J. Soileau, 2525 (1995).

J. Ramadas and R. Driver, Aspects of Secondary Students' Ideas About Light. Leeds, UK: University of Leeds, Centre for Studies in Science and Mathematics Education, (1989).

P. M. Sadler, "Students' Conceptions of Light and Color and How They Change", Bulletin of the American Astronomical Society, 32, p.1572 (2000).

M. H. Schneps and P. M. Sadler, Private Universe, Harvard Smithsonian Center for Astrophysics, (1989). see http://www.cfa.harvard.edu/education/k12.html

J. Stepans, Targeting Students' Science Misconceptions: Physical Science Activities Using the Conceptual Change Model (2nd edition). Riverview, Florida: Idea Factory, (1996). 